\title{
ON THE CONTROLLABILITY OF PARABOLIC SYSTEMS WITH A NONLINEAR TERM INVOLVING THE STATE AND THE GRADIENT*
}

\author{
A. DOUBOVA ${ }^{\dagger}$, E. FERNÁNDEZ-CARA ${ }^{\dagger}$, M. GONZÁLEZ-BURGOS ${ }^{\dagger}$, AND E. ZUAZUA $^{\ddagger}$
}

\begin{abstract}
We present some results concerning the controllability of a quasi-linear parabolic equation (with linear principal part) in a bounded domain of $\mathbb{R}^{N}$ with Dirichlet boundary conditions. We analyze the controllability problem with distributed controls (supported on a small open subset) and boundary controls (supported on a small part of the boundary). We prove that the system is null and approximately controllable at any time if the nonlinear term $f(y, \nabla y)$ grows slower than $|y| \log ^{3 / 2}(1+|y|+|\nabla y|)+|\nabla y| \log ^{1 / 2}(1+|y|+|\nabla y|)$ at infinity (generally, in this case, in the absence of control, blow-up occurs). The proofs use global Carleman estimates, parabolic regularity, and the fixed point method.
\end{abstract}

Key words. controllability, parabolic equations, nonlinear gradient terms

AMS subject classifications. 93B05, 35K55, 35K05

PII. S0363012901386465

1. Introduction and main results. Let $\Omega \subset \mathbb{R}^{N}$ be a bounded connected open set with boundary $\partial \Omega$ of class $C^{2}$. Let $\mathcal{O} \subset \Omega$ be a nonempty open subset, let $\gamma \subset \partial \Omega$ be a nonempty relative open subset of the boundary, and assume that $T>0$. We will use the following notation: $Q=\Omega \times(0, T), \Sigma=\partial \Omega \times(0, T)$. For any $p \in[1,+\infty]$, we will denote by $\|\cdot\|_{p}$ the usual norm in $L^{p}(Q)$.

We will consider parabolic systems of the form

$$
\begin{cases}\partial_{t} y-\Delta y+f(y, \nabla y)=v 1_{\mathcal{O}} & \text { in } \quad Q, \\ y=0 & \text { on } \Sigma, \\ y(x, 0)=y_{0}(x) & \text { in } \Omega\end{cases}
$$

and

$$
\left\{\begin{array}{lll}
\partial_{t} y-\Delta y+f(y, \nabla y)=0 & \text { in } & Q, \\
y=v 1_{\gamma} & \text { on } & \Sigma, \\
y(x, 0)=y_{0}(x) & \text { in } & \Omega,
\end{array}\right.
$$

where $y_{0}$ and $v$ are given in appropriate spaces. In (1) and (2),

$$
f: \mathbb{R} \times \mathbb{R}^{N} \rightarrow \mathbb{R}
$$

is a locally Lipschitz-continuous function and $1_{\mathcal{O}}$ and $1_{\gamma}$ denote the characteristic functions of the sets $\mathcal{O}$ and $\gamma$, respectively. We will assume that $y_{0} \in W^{1, \infty}(\Omega) \cap H_{0}^{1}(\Omega)$ (for simplicity), $v \in L^{\infty}(\mathcal{O} \times(0, T))$ in (1), and $v \in L^{\infty}(\gamma \times(0, T))$ in (2).

* Received by the editors March 14, 2001; accepted for publication (in revised form) January 17, 2002; published electronically August 8, 2002. This work was supported by grants PB96-0663, PB981134, and BFM2000-1317 of the DGES (Spain).

http://www.siam.org/journals/sicon/41-3/38646.html

${ }^{\dagger}$ Dpto. Ecuaciones Diferenciales y Análisis Numérico, Universidad de Sevilla, Aptdo. 1160, 41080 Sevilla, Spain (dubova@numer.us.es, cara@numer.us.es, burgos@numer.us.es).

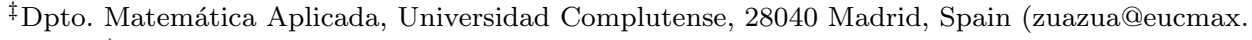
sim.ucm.es). 
The main goal of this paper is to analyze the controllability properties of (1) and (2). It will be said that (1) (resp., (2)) is null-controllable at time $T$ if, for each $y_{0} \in W^{1, \infty}(\Omega) \cap H_{0}^{1}(\Omega)$ (resp., $y_{0} \in W^{1, \infty}(\Omega) \cap V$, where $V$ is given below by (10)), there exists $v \in L^{\infty}\left(\mathcal{O} \times(0, T)\right.$ ) (resp., $v \in L^{\infty}(\gamma \times(0, T))$ ) such that the corresponding initial boundary problem (1) (resp., (2)) admits a solution $y \in$ $C^{0}\left([0, T] ; L^{2}(\Omega)\right)$ satisfying

$$
y(x, T)=0 \quad \text { in } \quad \Omega .
$$

On the other hand, it will be said that (1) (resp., (2)) is approximately controllable in $L^{2}(\Omega)$ at time $T$ if, for any $y_{0} \in W^{1, \infty}(\Omega) \cap H_{0}^{1}(\Omega)$ (resp., $y_{0} \in W^{1, \infty}(\Omega) \cap V$ ), any $y_{d} \in L^{2}(\Omega)$, and any $\varepsilon>0$, there exists a control $v \in L^{\infty}(\mathcal{O} \times(0, T)$ ) (resp., $\left.v \in L^{\infty}(\gamma \times(0, T))\right)$ such that the corresponding initial boundary problem (1) (resp., (2)) possesses a solution $y \in C^{0}\left([0, T] ; L^{2}(\Omega)\right)$, with

$$
\left\|y(\cdot, T)-y_{d}\right\|_{L^{2}} \leq \varepsilon .
$$

The controllability of linear and semilinear parabolic systems has been analyzed in several recent papers. Among them, let us mention $[\mathrm{I}],[\mathrm{FI}],[\mathrm{F}],[\mathrm{B}],[\mathrm{AB}]$, and [FZ2] in what concerns null controllability and [FPZ], [Z2], and [FZ2] for approximate controllability.

This paper generalizes all previous results, in particular those in [FZ2], where the nonlinear term is assumed to be of the form $f(y)$.

Notice that, under the hypothesis above, we can write

$$
f(s, p)=f(0,0)+g(s, p) s+G(s, p) \cdot p \quad \forall(s, p) \in \mathbb{R} \times \mathbb{R}^{N}
$$

for some $L_{\text {loc }}^{\infty}$ functions $g$ and $G$. These are respectively given by

$$
g(s, p)=\int_{0}^{1} \frac{\partial f}{\partial s}(\lambda s, \lambda p) d \lambda, \quad G_{i}(s, p)=\int_{0}^{1} \frac{\partial f}{\partial p_{i}}(\lambda s, \lambda p) d \lambda \quad \text { for } 1 \leq i \leq N .
$$

Our first result is the following one.

Theorem 1.1. Assume that $f$ is locally Lipschitz-continuous, $f(0,0)=0$ and

$$
\lim _{|(s, p)| \rightarrow \infty} \frac{|g(s, p)|}{\log ^{3 / 2}(1+|s|+|p|)}=0, \quad \lim _{|(s, p)| \rightarrow \infty} \frac{|G(s, p)|}{\log ^{1 / 2}(1+|s|+|p|)}=0 .
$$

Then (1) is null-controllable at any time $T>0$.

REMARK 1.1. This result generalizes at least two cases that have been studied exhaustively before. First, the case of a globally Lipschitz-continuous function $f$, i.e., when $g \in L^{\infty}\left(\mathbb{R} \times \mathbb{R}^{N}\right)$ and $G \in L^{\infty}\left(\mathbb{R} \times \mathbb{R}^{N}\right)^{N}$. In this case, $f$ is a function with sublinear behavior at infinity, and the proof of the corresponding controllability result is easier (cf. [IY]). Second, the case where $G \equiv 0$ and $g=g(s)$ satisfies $g(0)=0$ and

$$
\lim _{|s| \rightarrow \infty} \frac{|g(s)|}{\log ^{3 / 2}(1+|s|)}=0 .
$$

The proof is again easier (cf. [FZ2]).

REMARK 1.2. In [FZ2], it is proved that, for each $\beta>2$, there exist functions $f=f(s)$ with $f(0)=0$ and

$$
|f(s)| \sim|s| \log ^{\beta}(1+|s|) \quad \text { as } \quad|s| \rightarrow \infty
$$


such that (1) is not null-controllable for all $T>0$. In view of Theorem 1.1, we see that when $f$ satisfies (8) with $3 / 2 \leq \beta \leq 2$, the null controllability problem of (1) is an open question.

REMARK 1.3. Theorem 1.1 says in particular that, under assumption (6), for each $y_{0}$ there exists a control $v$ such that (1) possesses a solution globally defined in $[0, T]$. This claim is not true for any right-hand side and any $y_{0} \in W^{1, \infty}(\Omega) \cap H_{0}^{1}(\Omega)$, since we are in the range in which blow-up may occur (for instance, see $[\mathrm{CH}]$ ).

A consequence of Theorem 1.1 is the approximate controllability of (1). In this case, $f(0,0)$ will not be necessarily 0 and we will assume that $f$ verifies (5) and (9), a condition slightly different from (6). Thus, our second main result is the following.

Theorem 1.2. Let $T>0$. Assume that $f: \mathbb{R} \times \mathbb{R}^{N} \rightarrow \mathbb{R}$ is locally Lipschitzcontinuous and verifies

$$
\left\{\begin{array}{c}
\lim _{|(s, p)| \rightarrow \infty} \frac{1}{\log ^{3 / 2}(1+|s|+|p|)}\left|\int_{0}^{1} \frac{\partial f}{\partial s}\left(s_{0}+\lambda s, p_{0}+\lambda p\right) d \lambda\right|=0 \\
\lim _{|(s, p)| \rightarrow \infty} \frac{1}{\log ^{1 / 2}(1+|s|+|p|)}\left|\int_{0}^{1} \frac{\partial f}{\partial p_{i}}\left(s_{0}+\lambda s, p_{0}+\lambda p\right) d \lambda\right|=0
\end{array}\right.
$$

uniformly in $\left(s_{0}, p_{0}\right) \in K$ for every compact set $K \subset \mathbb{R} \times \mathbb{R}^{N}$. Then (1) is approximately controllable at time $T$.

REMARK 1.4. It will be seen in section 4 that, for systems like (1), the approximate controllability result is actually a consequence of the exact controllability to the trajectories in $C^{0}\left([0, T] ; W^{1, \infty}(\Omega)\right)$.

Remark 1.5. Again, Theorem 1.2 generalizes two known results. First, the case where $f$ is globally Lipschitz-continuous, i.e., the case in which $\partial f / \partial s$ and $\partial f / \partial p_{i}$ $(1 \leq i \leq N)$ are uniformly bounded (cf. [Z2]). On the other hand, Theorem 1.2 is also a generalization of the approximate controllability result in [FZ2], where $G \equiv 0$, $g=g(s)$, and (7) is satisfied.

In the first draft of this paper (and also in the approximate controllability results in [FZ2]), an additional assumption was imposed in Theorem 1.2, namely, the existence of a globally defined solution $y^{*}$ corresponding to appropriate data $y_{0}^{*}$ and $v^{*}$. But one of the referees provided an argument that shows that this hypothesis is in fact unnecessary (see the proof of Theorem 1.2 in section 4).

REMARK 1.6. In particular, (9) holds whenever $\partial f / \partial s$ and $\partial f / \partial p_{i}(1 \leq i \leq N)$ satisfy

$$
\lim _{|(s, p)| \rightarrow \infty} \frac{\left|\frac{\partial f}{\partial s}(s, p)\right|}{\log ^{3 / 2}(1+|s|+|p|)}=0, \quad \lim _{|(s, p)| \rightarrow \infty} \frac{\left|\frac{\partial f}{\partial p_{i}}(s, p)\right|}{\log ^{1 / 2}(1+|s|+|p|)}=0 .
$$

On the other hand, the assumptions (9) can be easily interpreted when $f=f(s)$. Indeed, in this case they simply read as follows:

$$
\left\{\begin{array}{l}
\lim _{|s| \rightarrow \infty} \frac{1}{\log ^{3 / 2}(1+|s|)}\left|\int_{0}^{1} f^{\prime}\left(s_{0}+\lambda s\right) d \lambda\right|=0 \\
\text { uniformly in } s_{0} \in K \text { for every compact set } K \subset \mathbb{R} .
\end{array}\right.
$$

The arguments in [FZ2] show that this is equivalent to (7) and also to

$$
\lim _{|s| \rightarrow \infty} \frac{1}{\log ^{3 / 2}(1+|s|)} \int_{0}^{1} f^{\prime}(\lambda s) d \lambda=0 .
$$


REMARK 1.7. It is proved in [FZ2] that, for each $\beta>2$, there exists a function $f=f(s)$ satisfying (8) such that the corresponding system (1) is not approximately controllable for all $T>0$. As in the case of null controllability, for $f$ satisfying (8) with $3 / 2 \leq \beta \leq 2$, the approximate controllability of (1) is an open question.

We can establish similar results for (2) under hypotheses of the same kind for $f$ and $y_{0}$. More precisely, let us introduce the Hilbert space

$$
V=\left\{z \in H^{1}(\Omega): z=0 \text { on } \partial \Omega \backslash \gamma\right\} .
$$

One has the following.

TheOREm 1.3. Let $T>0$. Assume that the assumptions in Theorem 1.1 are satisfied. Then (2) is null-controllable at any time $T>0$.

THEOREM 1.4. Let $T>0$. Assume that $f$ is locally Lipschitz-continuous and verifies (9). Then (2) is approximately controllable at time $T$.

REMARK 1.8. In the proofs of the previous controllability results, we will construct controls satisfying the appropriate properties. These controls are smooth. In particular, they will be such that the associated solutions of (1) and (2) belong to $C^{0}\left([0, T] ; W^{1, \infty}(\Omega)\right)$, a space where we can ensure uniqueness.

The rest of this paper is organized as follows. Section 2 is devoted to proving some technical lemmas we will use below. In section 3, we will prove Theorem 1.1. In section 4, we will give the proof of the approximate controllability result for system (1) (Theorem 1.2). Finally, the proofs of Theorems 1.3 and 1.4 will be sketched in section 5 .

2. Some technical results. Before giving the proofs of the theorems above, we have to present some technical results.

Let us consider the linear problem

$$
\begin{cases}\partial_{t} y-\Delta y+B \cdot \nabla y+a y=F & \text { in } \quad Q, \\ y=0 & \text { on } \Sigma, \\ y(x, 0)=y_{0}(x) & \text { in } \Omega,\end{cases}
$$

where $y_{0}$ and $F$ are given, $a \in L^{\infty}(Q)$, and $B \in L^{\infty}(Q)^{N}$. One has the following lemma, whose proof is essentially given in [LSU].

Lemma 2.1. Assume that $F \in L^{q}(Q)$ with $q>N+2, y_{0} \in W^{2, p}(\Omega) \cap H_{0}^{1}(\Omega)$ with $p>N, a \in L^{\infty}(Q)$, and $B \in L^{\infty}(Q)^{N}$. Then the solution $y$ of (11) satisfies

$$
\left\{\begin{array}{l}
y \in L^{q}\left(0, T ; W^{2, \beta}(\Omega)\right), \partial_{t} y \in L^{q}\left(0, T ; L^{\beta}(\Omega)\right), \\
\text { with } \quad \beta=\min (p, q)>N
\end{array}\right.
$$

and

$$
\left\{\begin{array}{c}
\|y\|_{L^{q}\left(0, T ; W^{2, \beta}\right)}+\left\|\partial_{t} y\right\|_{L^{q}\left(0, T ; L^{\beta}\right)} \\
\quad \leq C\left(\Omega, T,\|a\|_{\infty},\|B\|_{\infty}\right)\left(\left\|y_{0}\right\|_{W^{2, p}}+\|F\|_{q}\right) .
\end{array}\right.
$$

Furthermore, we also have $y \in C^{0}\left([0, T] ; W^{1, \infty}(\Omega)\right)$ and

$$
\|y\|_{C^{0}\left([0, T] ; W^{1, \infty}\right)} \leq M\left(\Omega, T,\|a\|_{\infty},\|B\|_{\infty}\right)\left(\left\|y_{0}\right\|_{W^{2, p}}+\|F\|_{q}\right),
$$

where

$$
\left\{\begin{array}{l}
M\left(\Omega, T,\|a\|_{\infty},\|B\|_{\infty}\right) \\
\quad=\exp \left[M_{0}\left(1+T+\left(T+T^{1 / 2}\right)\|a\|_{\infty}+\left(T+T^{1 / 2}\right)\|B\|_{\infty}^{2}\right)\right]
\end{array}\right.
$$


and $M_{0}$ is a positive constant depending only on $\Omega$.

For the reader's convenience, we have sketched the proof of this result in the appendix.

We will also recall a global Carleman inequality from [IY] for the linear problem

$$
\begin{cases}-\partial_{t} \varphi-\Delta \varphi=F_{0}+\sum_{i=1}^{N} \frac{\partial F_{i}}{\partial x_{i}} & \text { in } Q, \\ \varphi=0 & \text { on } \Sigma, \\ \varphi(x, T)=\varphi_{T}(x) & \text { in } \Omega,\end{cases}
$$

where $F_{0}, F_{i} \in L^{2}(Q)(1 \leq i \leq N)$ and $\varphi_{T} \in L^{2}(\Omega)$. One has the following.

LEMma 2.2. There exists a smooth function $\alpha_{0}=\alpha_{0}(x)$ that is defined and strictly positive for $x \in \bar{\Omega}$, and there exist positive constants $C_{0}$ and $\sigma_{0}$ (only depending on $\Omega$ and $\mathcal{O}$ ) such that

$$
\begin{gathered}
s^{3} \iint_{Q} e^{-2 s \alpha} t^{-3}(T-t)^{-3}|\varphi|^{2} \leq C_{0}\left(s^{3} \iint_{\mathcal{O} \times(0, T)} e^{-2 s \alpha} t^{-3}(T-t)^{-3}|\varphi|^{2}\right. \\
\left.+\iint_{Q} e^{-2 s \alpha}\left|F_{0}\right|^{2}+s^{2} \sum_{i=1}^{N} \iint_{Q} e^{-2 s \alpha} t^{-2}(T-t)^{-2}\left|F_{i}\right|^{2}\right)
\end{gathered}
$$

for all $s \geq s_{0}=\sigma_{0}\left(T+T^{2}\right)$, where $\varphi$ is the solution of (16) associated to $\varphi_{T} \in L^{2}(\Omega)$. In (17), the function $\alpha=\alpha(x, t)$ is given by

$$
\alpha(x, t)=\frac{\alpha_{0}(x)}{t(T-t)} .
$$

REMARK 2.1. The inequality (17) is based on a similar Carleman inequality for the heat equation with a right-hand side in $L^{2}(Q)$. The precise way $s_{0}$ depends on $T$ has been analyzed in [FZ1] and is essential in our analysis.

In what follows, unless otherwise specified, $C$ will stand for a generic positive constant depending only on $\Omega$ and $\mathcal{O}$, whose value can change from line to line. Let us introduce the following (adjoint) system:

$$
\begin{cases}-\partial_{t} q-\Delta q-\nabla \cdot(q B)+a q=0 & \text { in } Q, \\ q=0 & \text { on } \Sigma, \\ q(x, T)=q_{T}(x) & \text { in } \Omega,\end{cases}
$$

where $q_{T} \in L^{2}(\Omega)$. Arguing as in [FZ1], we can deduce from the Carleman estimates (17) an observability inequality for (18), as follows.

TheOREM 2.3. For any $a \in L^{\infty}(Q), B \in L^{\infty}(Q)^{N}$, and $q_{T} \in L^{2}(\Omega)$, one has

$$
\|q(\cdot, 0)\|_{L^{2}}^{2} \leq \exp \left[C B\left(T,\|a\|_{\infty},\|B\|_{\infty}\right)\right] \iint_{\mathcal{O} \times(0, T)}|q|^{2},
$$

where

$$
B\left(T,\|a\|_{\infty},\|B\|_{\infty}\right)=1+\frac{1}{T}+T\|a\|_{\infty}+\|a\|_{\infty}^{2 / 3}+(1+T)\|B\|_{\infty}^{2}
$$

and $q$ is the solution to the corresponding system (18). 
Proof. Let $a, B$, and $q_{T}$ be given and let $q$ be the solution to (18). Let us first see that

$$
\iint_{\Omega \times(T / 4,3 T / 4)}|q|^{2} \leq \exp \left[C\left(1+\frac{1}{T}+\|a\|_{\infty}^{2 / 3}+\|B\|_{\infty}^{2}\right)\right] \iint_{\mathcal{O} \times(0, T)}|q|^{2}
$$

We can write (17) for $\varphi=q$. This gives

$$
\begin{gathered}
s^{3} \iint_{Q} e^{-2 s \alpha} t^{-3}(T-t)^{-3}|q|^{2} \leq C_{0}\left(s^{3} \iint_{\mathcal{O} \times(0, T)} e^{-2 s \alpha} t^{-3}(T-t)^{-3}|q|^{2}\right. \\
\left.+\iint_{Q} e^{-2 s \alpha}|a q|^{2}+s^{2} \iint_{Q} e^{-2 s \alpha} t^{-2}(T-t)^{-2}|B q|^{2}\right)
\end{gathered}
$$

for all $s \geq s_{0}$. We can estimate the terms on the right as follows:

$$
\iint_{Q} e^{-2 s \alpha}|a q|^{2} \leq 2^{-6} T^{6}\|a\|_{\infty}^{2} \iint_{Q} e^{-2 s \alpha} t^{-3}(T-t)^{-3}|q|^{2}
$$

and

$$
\iint_{Q} e^{-2 s \alpha} t^{-2}(T-t)^{-2}|B q|^{2} \leq 2^{-2} T^{2}\|B\|_{\infty}^{2} \iint_{Q} e^{-2 s \alpha} t^{-3}(T-t)^{-3}|q|^{2} .
$$

Thus, we deduce from (21) that

$$
\iint_{Q} e^{-2 s \alpha} t^{-3}(T-t)^{-3}|q|^{2} \leq C \iint_{\mathcal{O} \times(0, T)} e^{-2 s \alpha} t^{-3}(T-t)^{-3}|q|^{2},
$$

provided

$$
s \geq s_{1}=\max \left(s_{0}, 2^{-4 / 3} C_{0}^{1 / 3} T^{2}\|a\|_{\infty}^{2 / 3}, C_{0} T^{2}\|B\|_{\infty}^{2}\right) .
$$

On the other hand, it can be easily verified that

$$
e^{-2 s \alpha} t^{-3}(T-t)^{-3} \leq 2^{6} T^{-6} \exp \left(-C s T^{-2}\right) \quad \forall(x, t) \in \bar{Q}
$$

and

$$
e^{-2 s \alpha} t^{-3}(T-t)^{-3} \geq\left(\frac{16}{3}\right)^{3} T^{-6} \exp \left(-C s T^{-2}\right) \quad \forall(x, t) \in \bar{\Omega} \times[T / 4,3 T / 4]
$$

whenever

$$
s \geq s_{2}=\max \left(s_{1}, 3 T^{2}\left(8 \min _{x \in \bar{\Omega}} \alpha_{0}(x)\right)^{-1}\right) .
$$

Analyzing the definitions of $s_{1}$ and $s_{2}$, we see that $s_{2} \leq s_{3}$, where $s_{3}$ is of the form

$$
s_{3}=\sigma_{3}\left(T+T^{2}+T^{2}\|a\|_{\infty}^{2 / 3}+T^{2}\|B\|_{\infty}^{2}\right)
$$

and $\sigma_{3}$ depends only on $\Omega$ and $\mathcal{O}$. From now on, we fix $s$, with $s=s_{3}$. Taking into account (23) and (24) and coming back to (22) (written for $s=s_{3}$ ), we deduce that (20) is satisfied for any solution $q$ of (18). 
Let us now prove that

$$
\|q(\cdot, T / 4)\|_{2}^{2} \leq \exp \left[C\left(\frac{1}{T}+T\|a\|_{\infty}+T\|B\|_{\infty}^{2}\right)\right] \iint_{\Omega \times(T / 4,3 T / 4)}|q|^{2} .
$$

Multiplying (18) by $q$ and integrating in $\Omega$, we obtain

$$
-\frac{1}{2} \frac{d}{d t} \int_{\Omega}|q|^{2} d x+\int_{\Omega}|\nabla q|^{2} d x=-\int_{\Omega} q B \cdot \nabla q d x-\int_{\Omega} a|q|^{2} d x \quad \forall t \geq 0 .
$$

Thus,

$$
-\frac{d}{d t} \int_{\Omega}|q|^{2} d x+\int_{\Omega}|\nabla q|^{2} d x \leq\left(\|B\|_{\infty}^{2}+2\|a\|_{\infty}\right) \int_{\Omega}|q|^{2} d x
$$

and

$$
\frac{d}{d t}\left(\exp \left(\left(2\|a\|_{\infty}+\|B\|_{\infty}^{2}\right) t\right) \int_{\Omega}|q|^{2} d x\right) \geq 0
$$

for all $t \geq 0$. Integrating this inequality with respect to the time variable in $[T / 4, t]$, where $t \in[T / 4,3 T / 4]$, we obtain

$$
\left\{\begin{aligned}
\int_{\Omega}|q(x, t)|^{2} d x & \geq \exp \left[\left(2\|a\|_{\infty}+\|B\|_{\infty}^{2}\right)(T / 4-t)\right] \int_{\Omega}|q(x, T / 4)|^{2} d x \\
& \geq \exp \left[-\left(\|a\|_{\infty}+\frac{1}{2}\|B\|_{\infty}^{2}\right) T\right] \int_{\Omega}|q(x, T / 4)|^{2} d x
\end{aligned}\right.
$$

for all $t \in[T / 4,3 T / 4]$. Integrating (27) again with respect to $t$, we find that

$$
\frac{T}{2} \int_{\Omega}|q(x, T / 4)|^{2} d x \leq \exp \left[\left(\|a\|_{\infty}+\frac{1}{2}\|B\|_{\infty}^{2}\right) T\right] \iint_{\Omega \times(T / 4,3 T / 4)}|q(x, t)|^{2},
$$

whence we easily deduce (25).

Finally, let us prove that

$$
\int_{\Omega}|q(x, 0)|^{2} d x \leq \exp \left[C T\left(\|a\|_{\infty}+\|B\|_{\infty}^{2}\right)\right] \int_{\Omega}|q(x, T / 4)|^{2} d x .
$$

This, together with (25) and (20), will lead to the desired observability estimate (19).

To prove (29), it suffices to integrate (26) in the time interval $[0, T / 4]$. Indeed, we find at once that

$$
\int_{\Omega}|q(x, 0)|^{2} d x \leq \exp \left[\left(2\|a\|_{\infty}+\|B\|_{\infty}^{2}\right) \frac{T}{4}\right] \int_{\Omega}|q(x, T / 4)|^{2} d x
$$

and thus (29) holds. This completes the proof of Theorem 2.3.

In fact, for the analysis of the controllability of (1) and (2), where $f$ is not necessarily globally Lipschitz-continuous, we need a refined version of the observability inequality (19). This is furnished by the following result.

Theorem 2.4. For any $a \in L^{\infty}(\Omega), B \in L^{\infty}(\Omega)^{N}$, and $q_{T} \in L^{2}(\Omega)$, one has

$$
\|q(\cdot, 0)\|_{L^{2}}^{2} \leq \exp \left[C K\left(T,\|a\|_{\infty},\|B\|_{\infty}\right)\right]\left(\iint_{\mathcal{O} \times(0, T)}|q|\right)^{2}
$$


where

$$
K\left(T,\|a\|_{\infty},\|B\|_{\infty}\right)=1+\frac{1}{T}+T+\left(T+T^{1 / 2}\right)\|a\|_{\infty}+\|a\|_{\infty}^{2 / 3}+(1+T)\|B\|_{\infty}^{2} .
$$

Proof. Let $\mathcal{O}^{\prime}$ be a nonempty open set such that $\mathcal{O}^{\prime} \subset \subset \mathcal{O}$. From Theorem 2.3 applied to $\mathcal{O}^{\prime}$ and the time interval $[T / 4,3 T / 4]$, we deduce that

$$
\|q(\cdot, T / 4)\|_{L^{2}}^{2} \leq \exp \left[C K^{\prime}\left(T,\|a\|_{\infty},\|B\|_{\infty}\right)\right] \iint_{\mathcal{O}^{\prime} \times(T / 4,3 T / 4)}|q|^{2},
$$

where $q$ is the solution of (18) associated to $q_{T} \in L^{2}(\Omega), K^{\prime}\left(T,\|a\|_{\infty},\|B\|_{\infty}\right)$ is given by

$$
K^{\prime}\left(T,\|a\|_{\infty},\|B\|_{\infty}\right)=1+\frac{1}{T}+T\|a\|_{\infty}+\|a\|_{\infty}^{2 / 3}+(1+T)\|B\|_{\infty}^{2},
$$

and $C$ is a new positive constant depending only on $\mathcal{O}^{\prime}$ (i.e., on $\mathcal{O}$ ) and $\Omega$. Using (26), we obtain

$$
\int_{\Omega}|q(x, 0)|^{2} d x \leq \exp \left[\frac{T}{4}\left(2\|a\|_{\infty}+\|B\|_{\infty}^{2}\right)\right] \int_{\Omega}|q(x, T / 4)|^{2} d x,
$$

and combining this with (32), we find that

$$
\|q(\cdot, 0)\|_{L^{2}}^{2} \leq \exp \left[C K^{\prime}\left(T,\|a\|_{\infty},\|B\|_{\infty}\right)\right] \iint_{\mathcal{O}^{\prime} \times(T / 4,3 T / 4)}|q|^{2} .
$$

At this point, we are going to use a technical result, related to the regularizing effect of the heat equation, whose proof will be given below.

LEMma 2.5. Let $\mathcal{O}_{i}, T_{i}, r_{i}$, and $\gamma_{i}(i=0,1)$ be given, with

$$
\left\{\begin{array}{l}
\mathcal{O}^{\prime} \subset \mathcal{O}_{0} \subset \subset \mathcal{O}_{1} \subset \mathcal{O}, \quad 0 \leq T_{1}<T_{0}<T / 2, \quad 1 \leq r_{1}<r_{0}<\infty, \\
1 \leq \gamma_{1}<\gamma_{0}<\infty, \quad \frac{1}{\gamma_{1}}-\frac{1}{\gamma_{0}}+\frac{N}{2}\left(\frac{1}{r_{1}}-\frac{1}{r_{0}}\right)<\frac{1}{2}
\end{array}\right.
$$

Then

$$
\begin{aligned}
& \left(\int_{T_{0}}^{T-T_{0}}\left(\int_{\mathcal{O}_{0}}|q|^{r_{0}} d x\right)^{\gamma_{0} / r_{0}} d t\right)^{1 / \gamma_{0}} \\
& \leq C T^{\lambda} H\left(T, T_{0}, T_{1},\|a\|_{\infty},\|B\|_{\infty}\right)\left(\int_{T_{1}}^{T-T_{1}}\left(\int_{\mathcal{O}_{1}}|q|^{r_{1}} d x\right)^{\gamma_{1} / r_{1}} d t\right)^{1 / \gamma_{1}}
\end{aligned}
$$

for all $q_{T} \in L^{2}(\Omega)$, with $C=C\left(\Omega, \mathcal{O}_{i}, r_{i}, \gamma_{i}, N\right), \lambda=\lambda\left(r_{i}, \gamma_{i}, N\right)$, and

$$
\left\{\begin{array}{l}
H\left(T, T_{0}, T_{1},\|a\|_{\infty},\|B\|_{\infty}\right) \\
\quad=1+\frac{T^{1 / 2}}{T_{0}-T_{1}}+T^{1 / 2}\left(1+\|a\|_{\infty}\right)+\left(1+T^{1 / 2}\right)\|B\|_{\infty} .
\end{array}\right.
$$

We will now apply this lemma together with (33). To this end, let us set $r_{0}=$ $\gamma_{0}=2$ and let us introduce the numbers $\gamma_{i}$ and $r_{i}$, given by the equalities

$$
\frac{1}{\gamma_{i}}=\frac{1}{r_{i}}=\frac{1}{2}+\frac{i}{2(N+2)}, \quad 1 \leq i \leq N+2 .
$$


It is immediate that $\gamma_{N+1}>1, r_{N+1}>1$, and $\gamma_{N+2}=r_{N+2}=1$. Now, let us set $\delta=T / 4(N+2)$. Accordingly,

$$
[T / 4-(N+2) \delta, 3 T / 4+(N+2) \delta]=[0, T] .
$$

Let us also introduce a family of open sets $\mathcal{O}_{i}$ such that

$$
\mathcal{O}^{\prime}=\mathcal{O}_{0} \subset \subset \mathcal{O}_{1} \subset \subset \mathcal{O}_{2} \subset \subset \cdots \subset \subset \mathcal{O}_{N+1} \subset \subset \mathcal{O}_{N+2}=\mathcal{O}
$$

For $0 \leq i \leq N+1$, we can use inequality (34) with $\mathcal{O}_{0}, \mathcal{O}_{1}, T_{0}, T_{1}, r_{0}, r_{1}, \gamma_{0}$, and $\gamma_{1}$, respectively, replaced by $\mathcal{O}_{i}, \mathcal{O}_{i+1}, T / 4-i \delta, T / 4-(i+1) \delta, r_{i}, r_{i+1}, \gamma_{i}$, and $\gamma_{i+1}$. The whole set of these inequalities gives

$$
\left(\iint_{\mathcal{O}^{\prime} \times(T / 4,3 T / 4)}|q|^{2}\right)^{1 / 2} \leq C T^{\alpha} H\left(T,\|a\|_{\infty},\|B\|_{\infty}\right)^{\beta}\left(\iint_{\mathcal{O} \times(0, T)}|q|\right)
$$

where $\beta=N+2$ and $\alpha$ is the sum of the exponents $\lambda_{i}$. If we now combine the inequalities (33) and (36), we obtain (30). This completes the proof of Theorem 2.4.

Proof of Lemma 2.5. Let $\rho_{1}$ and $\rho_{2}$ be functions in $\mathcal{D}\left(\mathcal{O}_{1}\right)$ and $\mathcal{D}\left(\left(T_{1}, T-T_{1}\right)\right)$, respectively, such that

$$
\rho_{1} \equiv 1 \text { in } \mathcal{O}_{0}, \quad \rho_{2} \equiv 1 \text { in }\left(T_{0}, T-T_{0}\right),
$$

and $0 \leq \rho_{1}, \rho_{2} \leq 1$. Let us put $\rho(x, t)=\rho_{1}(x) \rho_{2}(t)$ and $u=\rho q$, where $q$ is the solution to (18) associated to $q_{T} \in L^{2}(\Omega)$. Obviously,

$$
\operatorname{supp} u \subset \mathcal{O}_{1} \times\left(T_{1}, T-T_{1}\right)
$$

and

$$
\begin{cases}-\partial_{t} u-\Delta u=-a \rho q+\nabla \cdot(\rho q B)-\left(\partial_{t} \rho+\Delta \rho\right) q-2 \nabla \rho \cdot \nabla q-(\nabla \rho \cdot B) q & \text { in } Q \\ u=0 & \text { on } \Sigma, \\ u(x, T)=0 & \text { in } \Omega .\end{cases}
$$

In order to clarify the computations, let us put $\widetilde{u}(x, t)=u(x, T-t)$ for $(x, t) \in Q$. In a similar way, let us introduce the functions $\widetilde{a}, \widetilde{B}, \widetilde{\rho}$, and $\widetilde{q}$. We then have

$$
\begin{cases}\partial_{t} \widetilde{u}-\Delta \widetilde{u}=F & \text { in } Q, \\ \widetilde{u}=0 & \text { on } \Sigma, \\ \widetilde{u}(x, 0)=0 & \text { in } \Omega,\end{cases}
$$

where $F$ is given by

$$
F=-\widetilde{a} \widetilde{\rho} \widetilde{q}+\nabla \cdot(\widetilde{\rho} \widetilde{q} \widetilde{B})+\left(\partial_{t} \widetilde{\rho}-\Delta \widetilde{\rho}\right) \widetilde{q}-2 \nabla \widetilde{\rho} \cdot \nabla \widetilde{q}-(\nabla \widetilde{\rho} \cdot \widetilde{B}) \widetilde{q}
$$

Let us denote by $\{S(t): t \geq 0\}$ the semigroup generated by the heat equation with Dirichlet boundary conditions. Then one has

$$
\widetilde{u}(\cdot, t)=\int_{0}^{t} S(t-s) F(\cdot, s) d s
$$

where the integral can be understood, for instance, in $L^{r_{0}}(\Omega)$. 
Thanks to the regularizing effect of the heat equation, taking $L^{r_{0}}$-norms in (37), we obtain the following for $t \in\left(T_{1}, T-T_{1}\right)$ :

$$
\begin{gathered}
\|\widetilde{u}(\cdot, t)\|_{L^{r_{0}}} \leq C\left[\left(\|B\|_{\infty}+1\right) \int_{T_{1}}^{t}(t-s)^{-\frac{N}{2}\left(\frac{1}{r_{1}}-\frac{1}{r_{0}}\right)-\frac{1}{2}}\|\widetilde{q}(\cdot, s)\|_{L^{r_{1}}\left(\mathcal{O}_{1}\right)} d s\right. \\
\left.+\left(1+\frac{1}{T_{0}-T_{1}}+\|a\|_{\infty}+\|B\|_{\infty}\right) \int_{T_{1}}^{t}(t-s)^{-\frac{N}{2}\left(\frac{1}{r_{1}}-\frac{1}{r_{0}}\right)}\|\widetilde{q}(\cdot, s)\|_{L^{r_{1}}\left(\mathcal{O}_{1}\right)} d s\right] .
\end{gathered}
$$

Here, $C$ is a positive constant depending on $\mathcal{O}_{0}$ and $\mathcal{O}_{1}$. This gives

$$
\|\widetilde{u}(\cdot, t)\|_{L^{r_{0}}} \leq C H \int_{T_{1}}^{t}(t-s)^{-\frac{N}{2}\left(\frac{1}{r_{1}}-\frac{1}{r_{0}}\right)-\frac{1}{2}}\|\widetilde{q}(\cdot, s)\|_{L^{r_{1}}\left(\mathcal{O}_{1}\right)} d s
$$

for all $t \in\left(T_{1}, T-T_{1}\right)$, where $H=H\left(T, T_{0}, T_{1},\|a\|_{\infty},\|B\|_{\infty}\right)$ is given by (35). Due to the assumption

$$
\frac{N}{2}\left(\frac{1}{r_{1}}-\frac{1}{r_{0}}\right)+\frac{1}{\gamma_{1}}-\frac{1}{\gamma_{0}}<\frac{1}{2}
$$

we can apply Young's inequality to (39) and estimate the $L^{\gamma_{0}}\left(0, T ; L^{r_{0}}(\Omega)\right)$-norm of $\widetilde{u}$ as follows:

$$
\left(\int_{T_{1}}^{T-T_{1}}\|\widetilde{u}(\cdot, t)\|_{L^{r_{0}}}^{\gamma_{0}} d t\right)^{1 / \gamma_{0}} \leq C H T^{\lambda}\left(\int_{T_{1}}^{T-T_{1}}\|\widetilde{u}(\cdot, t)\|_{L^{r_{1}}\left(\mathcal{O}_{1}\right)}^{\gamma_{1}} d t\right)^{1 / \gamma_{1}}
$$

Here, $C$ is a new positive constant only depending on $\Omega, \mathcal{O}_{i}, r_{i}$, and $\gamma_{i}$, and $N$ and $H$ are given by (35) and

$$
\lambda=-\left[\frac{N}{2}\left(\frac{1}{r_{1}}-\frac{1}{r_{0}}\right)+\frac{1}{\gamma_{1}}-\frac{1}{\gamma_{0}}\right]+\frac{1}{2} .
$$

Inequality (34) is directly obtained from (40). This completes the proof of Lemma 2.5.

REMARK 2.2. As an easy consequence of Theorem 2.4 and (30), we can also deduce for each $r \in(1, \infty)$ an observability inequality in $L^{r}(\mathcal{O} \times(0, T))$ :

$$
\|q(\cdot, 0)\|_{L^{2}}^{2} \leq \exp \left[C_{r} K\left(T,\|a\|_{\infty},\|B\|_{\infty}\right)\right]\left(\iint_{\mathcal{O} \times(0, T)}|q|^{r}\right)^{\frac{2}{r}}
$$

for any $a \in L^{\infty}(\Omega), B \in L^{\infty}(\Omega)^{N}$, and $q_{T} \in L^{2}(\Omega)$. In (41), $K\left(T,\|a\|_{\infty},\|B\|_{\infty}\right)$ is given by (31) and $C_{r}$ only depends on $\Omega, \mathcal{O}$, and $r$.

3. Proof of the null controllability result. This section is devoted to proving Theorem 1.1. Using Theorem 2.4, we will first establish a null controllability result for a similar linear heat equation with controls in $L^{\infty}(\mathcal{O} \times(0, T))$. We will then apply a fixed point argument to obtain the desired result. The structure of the proof (the controllability of a similar linear system together with a fixed point argument) is rather general. It was introduced in [Z1] in the context of the boundary controllability of the semilinear wave equation. For other results proved in a similar way, see, for instance, $[\mathrm{FPZ}]$ and $[\mathrm{FI}]$. 
3.1. A null controllability result for a linear problem. We will consider the linear system

$$
\begin{cases}\partial_{t} y-\Delta y+B \cdot \nabla y+a y=v 1_{\mathcal{O}} & \text { in } \quad Q \\ y=0 & \text { on } \Sigma, \\ y(x, 0)=y_{0}(x) & \text { in } \quad \Omega\end{cases}
$$

where $a \in L^{\infty}(Q), B \in L^{\infty}(Q)^{N}$, and $y_{0} \in L^{2}(\Omega)$ are given. The following holds.

Theorem 3.1. Assume that $T>0, a \in L^{\infty}(Q), B \in L^{\infty}(Q)^{N}$, and $y_{0} \in L^{2}(\Omega)$. Then there exists a control $\widehat{v} \in L^{\infty}(\mathcal{O} \times(0, T))$ such that the corresponding solution of (42) satisfies

$$
\widehat{y}(x, T)=0 \quad \text { in } \Omega .
$$

Furthermore, $\widehat{v}$ can be chosen in such a way that

$$
\|\widehat{v}\|_{L^{\infty}(\mathcal{O} \times(0, T))} \leq \exp \left[C K\left(T,\|a\|_{\infty},\|B\|_{\infty}\right)\right]\left\|y_{0}\right\|_{L^{2}}
$$

where $K\left(T,\|a\|_{\infty},\|B\|_{\infty}\right)$ is given by (31).

Proof. For every $\varepsilon>0$, let us consider the functional $J_{\varepsilon}$, with

$$
J_{\varepsilon}\left(q_{T}\right)=\frac{1}{2}\left(\iint_{\mathcal{O} \times(0, T)}|q|\right)^{2}+\varepsilon\left\|q_{T}\right\|_{L^{2}}+\int_{\Omega} q(x, 0) y_{0}(x) d x \quad \forall q_{T} \in L^{2}(\Omega) .
$$

Here, $q$ is the solution of (18) associated to $q_{T} \in L^{2}(\Omega)$.

It is easy to see that $J_{\varepsilon}$ is a continuous and strictly convex functional in $L^{2}(\Omega)$. Furthermore, from (22), it is immediate to deduce the following unique continuation property for (18): If $q=0$ in $\mathcal{O} \times(0, T)$, then $q \equiv 0$.

Thus, arguing as in [FPZ], we also see that

$$
\liminf _{\left\|q_{T}\right\|_{L^{2}} \rightarrow \infty} \frac{J_{\varepsilon}\left(q_{T}\right)}{\left\|q_{T}\right\|_{L^{2}}} \geq \varepsilon
$$

and, therefore, $J_{\varepsilon}$ achieves its minimum at a unique point $\widehat{q}_{T}^{\varepsilon} \in L^{2}(\Omega)$.

Let $\widehat{q}_{\varepsilon}$ be the solution of (18) associated to $\widehat{q}_{T}^{\varepsilon}$. Taking $v=\widehat{v}_{\varepsilon}$ in (42) with

$$
\widehat{v}_{\varepsilon} \in\left(\operatorname{sgn} \widehat{q}_{\varepsilon}\right)\left(\int_{\mathcal{O} \times(0, T)}\left|\widehat{q}_{\varepsilon}\right|\right) 1_{\mathcal{O}}
$$

and arguing as in [FPZ], we see that the associated solution $\widehat{y}_{\varepsilon}$ satisfies

$$
\left\|\widehat{y}_{\varepsilon}(\cdot, T)\right\|_{L^{2}} \leq \varepsilon
$$

It is not difficult to see that

$$
\left\|\widehat{v}_{\varepsilon}\right\|_{L^{\infty}(\mathcal{O} \times(0, T))}=\iint_{\mathcal{O} \times(0, T)}\left|\widehat{q}_{\varepsilon}\right| \leq \exp \left[C K\left(T,\|a\|_{\infty},\|B\|_{\infty}\right)\right]\left\|y_{0}\right\|_{L^{2}}
$$

for all $\varepsilon>0$. Indeed, the fact that

$$
\left\|\widehat{v}_{\varepsilon}\right\|_{L^{\infty}(\mathcal{O} \times(0, T))}=\iint_{\mathcal{O} \times(0, T)}\left|\widehat{q}_{\varepsilon}\right|
$$


is implied by (46). On the other hand, since

$$
J_{\varepsilon}\left(\widehat{q}_{T}^{\varepsilon}\right) \leq J_{\varepsilon}(0)=0,
$$

we see from (45) that

$$
\frac{1}{2}\left(\iint_{\mathcal{O} \times(0, T)}\left|\widehat{q}_{\varepsilon}\right|\right)^{2} \leq-\int_{\Omega} \widehat{q}_{\varepsilon}(x, 0) y_{0}(x) d x \leq\left\|\widehat{q}_{\varepsilon}(\cdot, 0)\right\|_{L^{2}}\left\|y_{0}\right\|_{L^{2}} .
$$

In view of (30), (48) holds.

Since $\widehat{v}_{\varepsilon}$ is uniformly bounded in $L^{\infty}(\mathcal{O} \times(0, T))$, at least for an appropriate subsequence we must have

$$
\widehat{v}_{\varepsilon} \rightarrow \widehat{v} \quad \text { weakly-* in } L^{\infty}(\mathcal{O} \times(0, T)),
$$

where $\widehat{v} \in L^{\infty}(\mathcal{O} \times(0, T))$ satisfies (44). Accordingly,

$$
\widehat{y}_{\varepsilon}(T) \rightarrow \widehat{y}(T) \text { in } L^{2}(\Omega),
$$

where $\widehat{y}$ is the solution of (42) associated to $\widehat{v}$. Since we have (47) for all $\varepsilon>0$, (43) is satisfied. This ends the proof.

3.2. Proof of Theorem 1.1. We are now ready to prove Theorem 1.1. First, observe that we can assume in this theorem that $y_{0} \in W^{2, p}(\Omega) \cap H_{0}^{1}(\Omega)$, with $p>N$. Indeed, it suffices to set $v=0$ for $t \in[0, \delta]$ and to work in the time interval $[\delta, T]$, looking at $y(\cdot, \delta)$ as the initial state.

As we said above, a fixed point argument will be used. For convenience, it will be assumed in a first step that $g$ and $G$ are continuous.

3.2.1. The case in which $\boldsymbol{g}$ and $\boldsymbol{G}$ are continuous. Let $y_{0}$ be given in $W^{2, p}(\Omega) \cap H_{0}^{1}(\Omega)$ with $p>N$. We will assume that

$$
g \in C^{0}\left(\mathbb{R} \times \mathbb{R}^{N}\right), \quad G \in C^{0}\left(\mathbb{R} \times \mathbb{R}^{N}\right)^{N},
$$

and (6) is satisfied. It is then clear that, for each $\varepsilon>0$, there exists $C_{\varepsilon}>0$ such that

$$
|g(s, p)|^{2 / 3}+|G(s, p)|^{2} \leq C_{\varepsilon}+\varepsilon \log (1+|s|+|p|) \quad \forall(s, p) \in \mathbb{R} \times \mathbb{R}^{N} .
$$

Let us set $Z=C^{0}\left([0, T] ; W^{1, \infty}(\Omega)\right)$ and let $R>0$ be a constant whose value will be determined below. We will use the truncation functions $\mathrm{T}_{R}: \mathbb{R} \mapsto \mathbb{R}$ and $\mathbf{T}_{R}: \mathbb{R}^{N} \mapsto \mathbb{R}^{N}$, given as follows:

$$
\mathrm{T}_{R}(s)=\left\{\begin{array}{cc}
s & \text { if }|s| \leq R \\
R \operatorname{sgn}(s) & \text { otherwise }
\end{array}\right.
$$

and

$$
\mathbf{T}_{R}(p)=\left(\mathrm{T}_{R}\left(p_{i}\right)\right)_{1 \leq i \leq N} \quad \forall p \in \mathbb{R}^{N} .
$$

For each $z \in Z$, we will consider the corresponding linear systems
$(52)\left\{\begin{array}{l}\partial_{t} y-\Delta y+G\left(\mathrm{~T}_{R}(\right. \\ y=0 \\ y(x, 0)=y_{0}(x)\end{array}\right.$
in $Q$, on $\Sigma$, in $\Omega$. 
We are going to associate to $z$ a family $U(z)$ of $L^{\infty}$-controls which serve to drive the solutions to zero. Observe that (52) is of the form (42) with

$$
\left\{\begin{array}{l}
a=a_{z}=g\left(\mathrm{~T}_{R}(z), \mathbf{T}_{R}(\nabla z)\right) \in L^{\infty}(Q), \\
B=B_{z}=G\left(\mathrm{~T}_{R}(z), \mathbf{T}_{R}(\nabla z)\right) \in L^{\infty}(Q)^{N} .
\end{array}\right.
$$

Consequently, we can apply Theorem 3.1 to (52). In fact, we are going to apply this result in an adequate (eventually smaller) time interval $\left(0, T_{z}\right)$, where

$$
T_{z}=\min \left\{T,\left\|g\left(\mathrm{~T}_{R}(z), \mathbf{T}_{R}(\nabla z)\right)\right\|_{\infty}^{-2 / 3},\left\|g\left(\mathrm{~T}_{R}(z), \mathbf{T}_{R}(\nabla z)\right)\right\|_{\infty}^{-1 / 3}\right\} .
$$

This is a key point in our proof that will lead to appropriate estimates (this idea is taken from [FZ2]).

From Theorem 3.1, we directly deduce the existence of a control $\widehat{v}_{z} \in L^{\infty}(\mathcal{O} \times$ $\left.\left(0, T_{z}\right)\right)$ such that the solution of $(52)$ in $\Omega \times\left(0, T_{z}\right)$ with $v=\widehat{v}_{z}$ satisfies

$$
\widehat{y}_{z}\left(x, T_{z}\right)=0 \text { in } \Omega
$$

and, moreover,

$$
\left\|\widehat{v}_{z}\right\|_{L^{\infty}\left(\mathcal{O} \times\left(0, T_{z}\right)\right)} \leq \exp \left[C K\left(T_{z},\left\|a_{z}\right\|_{\infty},\left\|B_{z}\right\|_{\infty}\right)\right]\left\|y_{0}\right\|_{L^{2}} .
$$

( $K$ is given by (31) and $a_{z}$ and $B_{z}$ are given by (53).)

Let $\widetilde{v}_{z}$ and $\widetilde{y}_{z}$ be the extensions by zero of $\widehat{v}_{z}$ and $\widehat{y}_{z}$ to the whole cylinder $Q=\Omega \times(0, T)$. It is clear that $\widetilde{y}_{z}$ is the corresponding solution of (52) associated to $\widetilde{v}_{z}$ and

$$
\widetilde{y}_{z}(x, T)=0 \quad \text { in } \Omega .
$$

From the definition of $T_{z}$, we see that

$$
\left\|\widetilde{v}_{z}\right\|_{L^{\infty}(\mathcal{O} \times(0, T))} \leq \exp \left[C\left(1+\left\|a_{z}\right\|_{\infty}^{2 / 3}+\left\|B_{z}\right\|_{\infty}^{2}\right)\right]\left\|y_{0}\right\|_{L^{2}}
$$

where the positive constant $C$ now depends on $\Omega, \mathcal{O}$, and $T$.

On the other hand, from (50) and Lemma 2.1, we obtain that

$$
\widehat{y}_{z} \in C^{0}\left(\left[0, T_{z}\right] ; W^{1, \infty}(\Omega)\right)
$$

and

$$
\left\|\widehat{y}_{z}\right\|_{C^{0}\left(\left[0, T_{z}\right] ; W^{1, \infty}\right)} \leq M\left(\Omega, T_{z},\left\|a_{z}\right\|_{\infty},\left\|B_{z}\right\|_{\infty}\right)\left(\left\|y_{0}\right\|_{W^{2, p}}+\left\|\widehat{v}_{z}\right\|_{L^{\infty}\left(\mathcal{O} \times\left(0, T_{z}\right)\right)}\right)
$$

( $M$ is given by (15)). Taking into account once again the definition of $T_{z}$, the estimate (56), and the definition of $\widetilde{y}_{z}$, we find that $\widetilde{y}_{z} \in Z$ and

$$
\left\|\widetilde{y}_{z}\right\|_{Z} \leq \exp \left[C\left(1+\left\|a_{z}\right\|_{\infty}^{2 / 3}+\left\|B_{z}\right\|_{\infty}^{2}\right)\right]\left\|y_{0}\right\|_{W^{2, p}},
$$

where (again) $C=C(\Omega, \mathcal{O}, T)$.

The estimates (56) and (57) can be written in the form

$$
\left\|\widetilde{v}_{z}\right\|_{L^{\infty}(\mathcal{O} \times(0, T))} \leq C_{1}(\Omega, \mathcal{O}, T, z)\left\|y_{0}\right\|_{L^{2}}
$$


and

$$
\left\|\widetilde{y}_{z}\right\|_{Z} \leq C_{1}(\Omega, \mathcal{O}, T, z)\left\|y_{0}\right\|_{W^{2, p}}
$$

where

$$
C_{1}(\Omega, \mathcal{O}, T, z)=\exp \left[C\left(1+\left\|a_{z}\right\|_{\infty}^{2 / 3}+\left\|B_{z}\right\|_{\infty}^{2}\right)\right] .
$$

For any given $v \in L^{\infty}(\mathcal{O} \times(0, T))$, let $y_{v} \in Z$ be the solution of (52) in $Q$ with right-hand side $v$. (In order to simplify the notation, we omit the dependence on $z$.) With this notation in mind, let us now set for each $z \in Z$

$U(z)=\left\{v \in L^{\infty}(\mathcal{O} \times(0, T)): y_{v}(T)=0, \quad\|v\|_{L^{\infty}(\mathcal{O} \times(0, T))} \leq C_{1}(\Omega, \mathcal{O}, T, z)\left\|y_{0}\right\|_{L^{2}}\right\}$

and

$$
\Lambda(z)=\left\{y_{v}: v \in U(z), \quad\left\|y_{v}\right\|_{Z} \leq C_{1}(\Omega, \mathcal{O}, T, z)\left\|y_{0}\right\|_{W^{2, p}}\right\} .
$$

In this way, we have been able to introduce a set-valued mapping on $Z$

$$
z \mapsto \Lambda(z)
$$

We will prove that this mapping possesses at least one fixed point $y$. We will also prove that, for some $R$, every fixed point of $\Lambda$ verifies

$$
\|y\|_{Z} \leq R .
$$

Of course, this will imply the existence of a control $v \in L^{\infty}(\mathcal{O} \times(0, T))$ such that (1) has a solution satisfying (3).

Let us see that Kakutani's fixed point theorem can be applied to $\Lambda$. (For the statement and proof of this result, see [A, Chapter 9, pp. 119-126].) First, from (58) and (59), we deduce that $\Lambda(z)$ is, for every $z \in Z$, a nonempty set. Moreover, it is easy to check that $\Lambda(z)$ is a uniformly bounded closed convex subset of $Z$. Owing to the regularity hypothesis on $y_{0}$ and Lemma 2.1, we have (12) (here $\beta=p$ ) and the estimate

$$
\|y\|_{L^{\infty}\left(0, T ; W^{2, p}\right)}+\left\|\partial_{t} y\right\|_{L^{\infty}\left(0, T ; L^{p}\right)} \leq C\left(\Omega, \mathcal{O}, T, R,\left\|y_{0}\right\|_{\left.W^{2, p}\right)}\right.
$$

(where $C\left(\Omega, \mathcal{O}, T, R,\left\|y_{0}\right\|_{W^{2, p}}\right)$ is independent of $z$ ) for any $y \in \Lambda(z)$. Since $p>N$, we can apply well-known compactness results and conclude that there exists a compact set $K \subset Z$ (which depends on $R$ ) such that

$$
\Lambda(z) \subset K \quad \forall z \in Z
$$

(for instance, see $[\mathrm{S}]$ ).

Let us now prove that the mapping $z \mapsto \Lambda(z)$ is upper hemicontinuous, i.e., that the real-valued function

$$
z \in Z \mapsto \sup _{y \in \Lambda(z)}\langle\mu, y\rangle
$$

is upper semicontinuous for each bounded linear form $\mu \in Z^{\prime}$. In other words, let us see that

$$
B_{\alpha, \mu}=\left\{z \in Z: \sup _{y \in \Lambda(z)}\langle\mu, y\rangle \geq \alpha\right\}
$$


is a closed set of $Z$ for every $\alpha \in \mathbb{R}$ and every $\mu \in Z^{\prime}$. Thus, let $\left\{z_{n}\right\}$ be a sequence in $B_{\alpha, \mu}$ such that $z_{n} \rightarrow z$ in $Z$. Our aim is to prove that $z \in B_{\alpha, \mu}$. In view of the continuity hypothesis on $g$ and $G$, we have

$$
g\left(\mathrm{~T}_{R}\left(z_{n}\right), \mathbf{T}_{R}\left(\nabla z_{n}\right)\right) \rightarrow g\left(\mathrm{~T}_{R}(z), \mathbf{T}_{R}(\nabla z)\right) \quad \text { in } \quad L^{\infty}(Q)
$$

and

$$
G\left(\mathrm{~T}_{R}\left(z_{n}\right), \mathbf{T}_{R}\left(\nabla z_{n}\right)\right) \rightarrow G\left(\mathrm{~T}_{R}(z), \mathbf{T}_{R}(\nabla z)\right) \quad \text { in } \quad L^{\infty}(Q)^{N} .
$$

Since all sets $\Lambda\left(z_{n}\right)$ are compact and satisfy (63), we deduce that

$$
\alpha \leq \sup _{y \in \Lambda\left(z_{n}\right)}\langle\mu, y\rangle=\left\langle\mu, y_{n}\right\rangle
$$

for some $y_{n} \in \Lambda\left(z_{n}\right)$. From the definitions of $\Lambda\left(z_{n}\right)$ and $U\left(z_{n}\right)$, there must exist $v_{n} \in L^{\infty}(\mathcal{O} \times(0, T))$ such that

$$
\partial_{t} y_{n}-\Delta y_{n}+G\left(\mathrm{~T}_{R}\left(z_{n}\right), \mathbf{T}_{R}\left(\nabla z_{n}\right)\right) \cdot \nabla y_{n}+g\left(\mathrm{~T}_{R}\left(z_{n}\right), \mathbf{T}_{R}\left(\nabla z_{n}\right)\right) y_{n}=v_{n} 1_{\mathcal{O}}
$$

in $Q$. Furthermore,

$$
\left\|v_{n}\right\|_{L^{\infty}(\mathcal{O} \times(0, T))} \leq C_{1}\left(\Omega, \mathcal{O}, T, z_{n}\right)\left\|y_{0}\right\|_{L^{2}}
$$

and

$$
\left\|y_{n}\right\|_{Z} \leq C_{1}\left(\Omega, \mathcal{O}, T, z_{n}\right)\left\|y_{0}\right\|_{W^{2, p}}
$$

whence $y_{n}$ (resp., $\left.v_{n}\right)$ is uniformly bounded in $Z$ (resp., $\left.L^{\infty}(\mathcal{O} \times(0, T))\right)$. Therefore, we can write the following at least for a subsequence:

$$
y_{n} \rightarrow \widehat{y} \quad \text { strongly in } Z
$$

(recall that (63) is satisfied) and

$$
v_{n} \rightarrow \widehat{v} \quad \text { weakly-* in } L^{\infty}(\mathcal{O} \times(0, T)) .
$$

Now, it is not difficult to check that

$$
\begin{cases}\partial_{t} \widehat{y}-\Delta \widehat{y}+G\left(\mathrm{~T}_{R}(z), \mathbf{T}_{R}(\nabla z)\right) \cdot \nabla \widehat{y}+g\left(\mathrm{~T}_{R}(z), \mathbf{T}_{R}(\nabla z)\right) \widehat{y}=\widehat{v} 1_{\mathcal{O}} & \text { in } \quad Q, \\ \widehat{y}=0 & \text { on } \Sigma, \\ \widehat{y}(x, 0)=y_{0}(x), \quad \widehat{y}(x, T)=0 & \text { in } \Omega,\end{cases}
$$

i.e., that $\widehat{v} \in U(z)$ and $\widehat{y} \in \Lambda(z)$. Consequently, we can take limits in (64) and deduce that

$$
\alpha \leq\langle\mu, \widehat{y}\rangle \leq \sup _{y \in \Lambda(z)}\langle\mu, y\rangle
$$

that is to say, $z \in B_{\alpha, \mu}$. This proves that $z \mapsto \Lambda(z)$ is upper hemicontinuous.

As a consequence, for any fixed $R>0$ Kakutani's theorem can be applied, ensuring the existence of a fixed point of $\Lambda$. As we said above, we will finish the proof by showing that we can choose $R>0$ in such a way that any fixed point of $\Lambda$ satisfies (62). It is just here where the assumptions (6) (in fact (51)) will be used. 
Thus, let $y$ be a fixed point of $\Lambda$ associated to the control $v \in U(y)$. Then (59), (60), and (51) lead to the estimates

$$
\begin{aligned}
\|y\|_{Z} & \leq \exp \left(C\left(1+\left\|g\left(\mathrm{~T}_{R}(y), \mathbf{T}_{R}(\nabla y)\right)\right\|_{\infty}^{2 / 3}+\left\|G\left(\mathrm{~T}_{R}(y), \mathbf{T}_{R}(\nabla y)\right)\right\|_{\infty}^{2}\right)\right)\left\|y_{0}\right\|_{W^{2, p}} \\
& \leq \exp \left(C\left(1+C_{\varepsilon}+\varepsilon \log (1+2 R)\right)\right)\left\|y_{0}\right\|_{W^{2, p}} \\
& =\exp \left(C\left(1+C_{\varepsilon}\right)\right)(1+2 R)^{C \varepsilon}\left\|y_{0}\right\|_{W^{2, p}}
\end{aligned}
$$

where $C=C(\Omega, \mathcal{O}, T)$. Taking $\varepsilon=1 /(2 C)$, we find that

$$
\|y\|_{Z} \leq C(1+2 R)^{1 / 2}\left\|y_{0}\right\|_{W^{2, p}},
$$

whence (62) holds whenever $R$ is large enough (depending on $\Omega, \mathcal{O}, T, g$, and $G$ ). We have then proved Theorem 1.1 in the case of smooth data.

3.2.2. The general case. Let us now suppose that $f$ is a locally Lipschitzcontinuous function satisfying assumption (5) (with $f(0,0)=0$ ) and (6). Let us introduce a function $\rho \in \mathcal{D}\left(\mathbb{R} \times \mathbb{R}^{N}\right)$ such that $\rho \geq 0$ in $\mathbb{R} \times \mathbb{R}^{N}$, supp $\rho \subset \bar{B}(0,1)$, and

$$
\iint_{\mathbb{R} \times \mathbb{R}^{N}} \rho(s, p) d s d p=1 .
$$

We consider the functions $\rho_{n}, g_{n}$, and $G_{n}(n \geq 1)$, with

$$
\begin{gathered}
\rho_{n}(s, p)=n^{N+1} \rho(n s, n p) \quad \forall(s, p) \in \mathbb{R} \times \mathbb{R}^{N}, \\
g_{n}=\rho_{n} * g, \quad G_{n}=\rho_{n} * G .
\end{gathered}
$$

Then it is not difficult to check that the following properties of $g_{n}$ and $G_{n}$ hold:

1. $g_{n} \in C^{0}\left(\mathbb{R} \times \mathbb{R}^{N}\right)$ and $G_{n} \in C^{0}\left(\mathbb{R} \times \mathbb{R}^{N}\right)^{N}$ for all $n \geq 1$.

2. If we put $f_{n}(s, p)=g_{n}(s, p) s+G_{n}(s, p) \cdot p$ for all $(s, p) \in \mathbb{R} \times \mathbb{R}^{N}$, then

$$
f_{n} \rightarrow f \quad \text { uniformly in the compact sets of } \mathbb{R} \times \mathbb{R}^{N} \text {. }
$$

3. For any given $M>0$, there exists $C(M)>0$ such that

$$
\sup _{|(s, p)| \leq M}\left(\left|g_{n}(s, p)\right|+\left|G_{n}(s, p)\right|\right) \leq C(M) \quad \forall n \geq 1 .
$$

4. The functions $g_{n}$ and $G_{n}$ verify (6) uniformly in $n$, that is to say, for any $\varepsilon>0$, there exists $M(\varepsilon)>0$ such that

$$
\left\{\begin{aligned}
\left|g_{n}(s, p)\right| & \leq \varepsilon \log ^{3 / 2}(1+|s|+|p|), \\
\left|G_{n}(s, p)\right| & \leq \varepsilon \log ^{1 / 2}(1+|s|+|p|)
\end{aligned}\right.
$$

whenever $|(s, p)| \geq M(\varepsilon)$ for all $n \geq 1$.

For every $n$, we can argue as in section 3.2.1 and find a control $v_{n} \in L^{\infty}(\mathcal{O} \times(0, T))$ such that the system

$$
\begin{cases}\partial_{t} y_{n}-\Delta y_{n}+f_{n}\left(y_{n}, \nabla y_{n}\right)=v_{n} 1_{\mathcal{O}} & \text { in } Q \\ y_{n}=0 & \text { on } \Sigma \\ y_{n}(x, 0)=y_{0}(x) & \text { in } \Omega\end{cases}
$$


possesses at least one solution $y_{n} \in Z$ satisfying

$$
y_{n}(x, T)=0 \quad \text { in } \Omega .
$$

From the properties satisfied by $g_{n}$ and $G_{n}$, and thanks to the estimates obtained in section 3.2.1, we deduce that

$$
\left\|v_{n}\right\|_{L^{\infty}(\mathcal{O} \times(0, T))} \leq C \quad \text { and } \quad\left\|y_{n}\right\|_{Z} \leq C
$$

for all $n \geq 1$. In fact, in view of Lemma 2.1 we have $y_{n} \in K$ for all $n$, where $K$ is a fixed compact set in $Z$. Accordingly, we can assume that, at least for a subsequence,

$$
v_{n} \rightarrow v \quad \text { weakly-* in } L^{\infty}(\mathcal{O} \times(0, T))
$$

and

$$
y_{n} \rightarrow y \quad \text { strongly in } Z \text {. }
$$

Hence, passing to the limit in (66), we find a control $v \in L^{\infty}(\mathcal{O} \times(0, T))$ such that (1) possesses a solution $y$ satisfying (3). This ends the proof of Theorem 1.1.

REMARK 3.1. Analyzing the proof of Theorem 1.1, we deduce that the null controllability result remains valid if we change (6) by the following assumptions:

$$
\limsup _{|(s, p)| \rightarrow \infty} \frac{|g(s, p)|}{\log ^{3 / 2}(1+|s|+|p|)} \leq l_{1}<\infty, \quad \limsup _{|(s, p)| \rightarrow \infty} \frac{|G(s, p)|}{\log ^{1 / 2}(1+|s|+|p|)} \leq l_{2}<\infty
$$

where $l_{1}$ and $l_{2}$ are positive and sufficiently small (depending only on $\Omega$ and $\mathcal{O}$ ).

REMARK 3.2. In Theorem 1.1, we can consider as well a more general nonlinear term of the form $f(x, t ; s, p)$, with $(x, t) \in Q$ and $(s, p) \in \mathbb{R} \times \mathbb{R}^{N}$. The assumptions on $f$ have to be the following in this case:

1. $f(x, t ; 0,0)=0$ for all $(x, t) \in Q$,

2. $f(\cdot ; s, p) \in L^{\infty}(Q)$ for all $(s, p) \in \mathbb{R} \times \mathbb{R}^{N}$,

3. $f(x, t ; \cdot)$ is locally Lipschitz-continuous for $(x, t)$ a.e. in $Q$, with Lipschitz constants independent of $(x, t)$ in the bounded sets of $\mathbb{R} \times \mathbb{R}^{N}$,

4. $f(\cdot ; s, p)=g(\cdot ; s, p) s+G(\cdot ; s, p) \cdot p$ for all $(s, p) \in \mathbb{R} \times \mathbb{R}^{N}$, with

$$
\lim _{|(s, p)| \rightarrow \infty} \frac{|g(x, t ; s, p)|}{\log ^{3 / 2}(1+|s|+|p|)}=0, \quad \lim _{|(s, p)| \rightarrow \infty} \frac{|G(x, t ; s, p)|}{\log ^{1 / 2}(1+|s|+|p|)}=0
$$

uniformly in $(x, t) \in Q$.

REMARK 3.3. Adapting the arguments used in the proof of Theorem 1.1, we can deduce a local null controllability result for (1) with a general nonlinear term $f(s, p)$ satisfying $f(0,0)=0$. To be precise, if $f$ is given, there exists $\delta=\delta(\Omega, \mathcal{O}, T, f)>0$ such that for every $y_{0} \in W^{2, p}(\Omega) \cap H_{0}^{1}(\Omega)(p>N)$ with $\left\|y_{0}\right\|_{W^{2, p}} \leq \delta$, a control $v \in L^{\infty}(\mathcal{O} \times(0, T))$ can be found such that the corresponding problem has a unique solution $y \in L^{\infty}\left(0, T ; W^{1, \infty}(\Omega)\right)$ which satisfies

$$
y(x, T)=0 \quad \text { in } \Omega .
$$


4. Proof of the approximate controllability result. In this section we will prove Theorem 1.2. Let us fix $T>0, \varepsilon>0, y_{0} \in W^{1, \infty}(\Omega) \cap H_{0}^{1}(\Omega)$, and $y_{d} \in$ $W^{2, p}(\Omega) \cap H_{0}^{1}(\Omega)$ with $p>N$ (for instance). Obviously, it will be sufficient to consider final data in $W^{2, p}(\Omega) \cap H_{0}^{1}(\Omega)$, since this space is dense in $L^{2}(\Omega)$. We will present the proof in several steps and start with a result concerning the exact controllability to the trajectories in $C^{0}\left([0, T] ; W^{1, \infty}(\Omega)\right)$.

Lemma 4.1. Assume the hypotheses on $f$ in Theorem 1.2 are satisfied. Let $y_{0} \in W^{1, \infty}(\Omega) \cap H_{0}^{1}(\Omega)$ be given and let $y^{*}$ be a solution to $(1)$ in $C^{0}\left([0, T] ; W^{1, \infty}(\Omega)\right)$ corresponding to the data

$$
y_{0}^{*} \in W^{1, \infty}(\Omega) \cap H_{0}^{1}(\Omega), \quad v^{*} \in L^{\infty}(\mathcal{O} \times(0, T)) .
$$

There exists a control $v \in L^{\infty}(\mathcal{O} \times(0, T))$ and a state $y \in C^{0}\left([0, T] ; W^{1, \infty}(\Omega)\right)$ associated to $y_{0}$ and $v$ such that

$$
y(x, T)=y^{*}(x, T) \quad \text { in } \quad \Omega .
$$

Proof. Let us put $y=y^{*}+w$. We will look for a control $u \in L^{\infty}(\mathcal{O} \times(0, T))$ such that the solution of

$$
\begin{cases}\partial_{t} w-\Delta w+F(x, t ; w, \nabla w)=u 1_{\mathcal{O}} & \text { in } Q, \\ w=0 & \text { on } \Sigma, \\ w(0)=y_{0}-y_{0}^{*} & \text { in } \Omega\end{cases}
$$

satisfies

$$
w(x, T)=0 \quad \text { in } \quad \Omega .
$$

Here, $F$ is given by

$$
F(x, t ; s, p)=f\left(y^{*}(x, t)+s, \nabla y^{*}(x, t)+p\right)-f\left(y^{*}(x, t), \nabla y^{*}(x, t)\right)
$$

for all $(x, t) \in Q$ and $(s, p) \in \mathbb{R} \times \mathbb{R}^{N}$. The proof of this lemma will be achieved if we check that such a control $u$ exists.

Notice that

$$
F(x, t ; s, p)=\widetilde{g}(x, t ; s, p) s+\widetilde{G}(x, t ; s, p) \cdot p,
$$

where

$$
\widetilde{g}(x, t ; s, p)=\int_{0}^{1} \frac{\partial f}{\partial s}\left(y^{*}(x, t)+\lambda s, \nabla y^{*}(x, t)+\lambda p\right) d \lambda
$$

and

$$
\widetilde{G}_{i}(x, t ; s, p)=\int_{0}^{1} \frac{\partial f}{\partial p_{i}}\left(y^{*}(x, t)+\lambda s, \nabla y^{*}(x, t)+\lambda p\right) d \lambda \quad \text { for } 1 \leq i \leq N .
$$

Thus, in view of (9) and the fact that $y^{*} \in C^{0}\left([0, T] ; W^{1, \infty}(\Omega)\right)$, it is clear that $F$ satisfies the assumptions of Remark 3.2. This is sufficient to ensure that $u$ exists. This completes the proof of this lemma.

Now, we argue as follows: 
- There exists $\delta_{0}>0$, depending only on $\Omega, y_{d}$, and $f$, such that the system

$$
\begin{cases}\partial_{t} w-\Delta w+f(w, \nabla w)=0 & \text { in } \Omega \times\left(0, \delta_{0}\right), \\ w=0 & \text { on } \partial \times\left(0, \delta_{0}\right), \\ w(x, 0)=y_{d}(x) & \text { in } \Omega\end{cases}
$$

has exactly one solution $w \in C^{0}\left(\left[0, \delta_{0}\right] ; W^{1, \infty}(\Omega)\right)$ also satisfying

$$
w(\cdot, t) \in W^{2, p}(\Omega) \cap H_{0}^{1}(\Omega) \quad \forall t \in\left[0, \delta_{0}\right] .
$$

Obviously, we can associate to $\varepsilon$ a parameter $\delta_{1} \in\left(0, \delta_{0}\right]$ (small enough) such that

$$
\left\|w(\cdot, t)-y_{d}\right\|_{L^{2}} \leq \varepsilon \quad \forall t \in\left[0, \delta_{1}\right] .
$$

In what follows, we fix $\delta_{1}$ verifying $(70)$.

- There exists $v_{1} \in L^{\infty}\left(\mathcal{O} \times\left(0, \delta_{1}\right)\right)$ such that the corresponding system

$$
\begin{cases}\partial_{t} y-\Delta y+f(y, \nabla y)=v_{1} 1_{\mathcal{O}} & \text { in } \Omega \times\left(0, \delta_{1}\right), \\ y=0 & \text { on } \partial \Omega\left(0, \delta_{1}\right), \\ y(x, 0)=y_{0}(x) & \text { in } \Omega\end{cases}
$$

possesses exactly one solution $y_{1} \in C^{0}\left(\left[0, \delta_{1}\right] ; W^{1, \infty}(\Omega)\right)$, with

$$
y_{1}\left(x, \delta_{1}\right)=w\left(x, \delta_{1}\right) \text { in } \Omega .
$$

This is a consequence of Lemma 4.1.

- On the other hand, there exists $\widetilde{v} \in L^{\infty}\left(\mathcal{O} \times\left(0, \delta_{1}\right)\right)$ such that the system

$$
\begin{cases}\partial_{t} y-\Delta y+f(y, \nabla y)=\widetilde{v} 1_{\mathcal{O}} & \text { in } \quad \Omega \times\left(0, \delta_{1}\right), \\ y=0 & \text { on } \partial \Omega\left(0, \delta_{1}\right), \\ y(x, 0)=w\left(x, \delta_{1}\right) & \text { in } \Omega\end{cases}
$$

possesses exactly one solution $\widetilde{y} \in C^{0}\left(\left[0, \delta_{1}\right] ; W^{1, \infty}(\Omega)\right)$, with

$$
\widetilde{y}\left(x, \delta_{1}\right)=w\left(x, \delta_{1}\right) \text { in } \Omega .
$$

This is again a consequence of Lemma 4.1.

- Assume that $T=n \delta_{1}+\delta$ for some integer $n \geq 0$ and some $\delta \in\left[0, \delta_{1}\right)$. Let us put $I_{k}=\left[k \delta_{1},(k+1) \delta_{1}\right)$ for $0 \leq k \leq n-1$ and $I_{n}=\left[n \delta_{1}, T\right]$. We will construct the control $v$ as follows.

For $t \in I_{0}$, we set $v(x, t)=v_{1}(x, t)$ a.e., where $v_{1}$ is the control arising in (71). Then, for $1 \leq k \leq n-1$ and $t \in I_{k}$, we set $v(x, t)=\widetilde{v}\left(x, t-k \delta_{1}\right)$, where $\widetilde{v}$ is the control in (72).

If $\delta=0$, we have constructed in this way a control $v \in L^{\infty}(\mathcal{O} \times(0, T))$ such that the associate state $y$ satisfies

$$
y(x, T)=w\left(x, \delta_{1}\right) \text { in } \Omega .
$$

In view of (70), (4) is satisfied.

If $\delta \in\left(0, \delta_{1}\right)$, then we complete the definition of $v$ by setting $v(x, t)=\widehat{v}\left(x, t-n \delta_{1}\right)$ for all $t \in I_{n}$. Here, $\widehat{v} \in L^{\infty}(\mathcal{O} \times(0, \delta))$ is a control such that the system

$$
\begin{cases}\partial_{t} y-\Delta y+f(y, \nabla y)=\widehat{v} 1_{\mathcal{O}} & \text { in } \Omega \times(0, \delta), \\ y=0 & \text { on } \partial \Omega \times(0, \delta), \\ y(x, 0)=w\left(x, \delta_{1}\right) & \text { in } \Omega\end{cases}
$$


possesses exactly one solution $\hat{y} \in C^{0}\left([0, \delta] ; W^{1, \infty}(\Omega)\right)$ satisfying

$$
\hat{y}(x, \delta)=w(x, \delta) \quad \text { in } \quad \Omega .
$$

(Once more, the existence of $\hat{v}$ is implied by Lemma 4.1.) Now, the state $y$ associated to $y_{0}$ and $v$ satisfies

$$
y(x, T)=w(x, \delta) \text { in } \Omega .
$$

Again, taking (70) into account, we see that (4) is satisfied in this case.

This completes the proof of Theorem 1.2.

5. Sketch of the proofs of the boundary controllability results. We devote this section to sketching briefly the proofs of Theorems 1.3 and 1.4. Both results are implied by the results established in the case of internal controllability.

For instance, let us refer to the proof of Theorem 1.3. Let us assume, for simplicity, that $y_{0} \in W^{2, p}(\Omega) \cap V$ for some $p>N$ (recall that $V$ is given by (10)). We have assumed that $f: \mathbb{R} \times \mathbb{R}^{N} \mapsto \mathbb{R}$ is a locally Lipschitz-continuous function that satisfies $f(0,0)=0$ and (6). Let $D$ be a bounded open set with boundary $\partial D$ of class $C^{2}$ such that $\Omega \subset D$ and $\partial \Omega \cap D=\gamma$. Let $\mathcal{O}$ be an open subset of $D \backslash \bar{\Omega}$. There exists a function $\widetilde{y}_{0} \in W^{2, p}(D) \cap H_{0}^{1}(D)$ such that $\widetilde{y}_{0}=y_{0}$ in $\Omega$ and

$$
\left\|\widetilde{y}_{0}\right\|_{W^{2, p}(D)} \leq C\left\|y_{0}\right\|_{W^{2, p}(\Omega)},
$$

where $C$ is a positive constant depending only on $\Omega$ and $D$.

Let $\widetilde{v} \in L^{\infty}(\mathcal{O} \times(0, T))$ be a control, furnished by Theorem 1.1, such that

$$
\left\{\begin{array}{lll}
\partial_{t} \widetilde{y}-\Delta \widetilde{y}+f(\widetilde{y}, \nabla \widetilde{y})=\widetilde{v} 1_{\mathcal{O}} & \text { in } & D \times(0, T), \\
\widetilde{y}=0 & \text { on } & \partial D \times(0, T), \\
\widetilde{y}(x, 0)=\widetilde{y}_{0}(x) & \text { in } & D
\end{array}\right.
$$

possesses exactly one solution $\widetilde{y} \in C^{0}\left([0, T] ; W^{1, \infty}(D)\right)$ with

$$
\widetilde{y}(x, T)=0 \quad \text { in } D .
$$

Let $v$ be the trace of $\widetilde{y}$ on $\gamma \times(0, T)$. Then $v \in L^{\infty}(\gamma \times(0, T))$, and the restriction to $\Omega \times(0, T)$ of $\widetilde{y}$ solves the corresponding system (2). This proves Theorem 1.3.

In order to prove Theorem 1.4, it suffices to argue in a similar way.

Appendix. Proof of Lemma 2.1. The statement (12) and the inequality (13) are proved in [LSU, Theorem 9.1, p. 342]. The inequality (14) is not explicitly proved in [LSU], but it can be deduced (in several ways) from other results of this book. One of the arguments is as follows.

From Theorem 16.3 in [LSU] (p. 412), we deduce the inequalities

$$
\|S(t) \varphi\|_{L^{\gamma}} \leq C t^{-\frac{N}{2}\left(\frac{1}{r}-\frac{1}{\gamma}\right)}\|\varphi\|_{L^{r}}
$$

and

$$
\|S(t) \varphi\|_{W^{1, \gamma}} \leq C t^{-\frac{N}{2}\left(\frac{1}{r}-\frac{1}{\gamma}\right)-\frac{1}{2}}\|\varphi\|_{L^{r}},
$$

which hold for all $t>0$ and $r, \gamma \in[1, \infty]$ with $\gamma \geq r$. Here, $S(t)$ is the semigroup generated by the heat equation with Dirichlet boundary conditions. With these inequalities in mind, one can prove the following. 
Lemma A.1. Let $y$ be the solution of (11). If $y \in L^{\infty}\left(0, T ; W^{1, r}(\Omega)\right)$ with $r \leq 2 N$, then

$$
y \in L^{\infty}\left(0, T ; W^{1, \gamma}(\Omega)\right), \quad \text { where } \gamma=\left\{\begin{array}{cl}
\left(\frac{1}{r}-\frac{1}{2 N}\right)^{-1} & \text { if } r<2 N \\
\infty & \text { if } r=2 N
\end{array}\right.
$$

and

$$
\left\{\begin{aligned}
\|y\|_{L^{\infty}\left(0, T ; W^{1, \gamma}\right)} \leq & e^{C(1+T)}\left(\left\|y_{0}\right\|_{W^{2, p}}+\|F\|_{q}\right) \\
& +e^{C\left(1+T^{1 / 2}\|B\|_{\infty}^{2}+T^{1 / 2}\|a\|_{\infty}\right)}\|y\|_{L^{\infty}\left(0, T ; W^{1, r}\right)} .
\end{aligned}\right.
$$

Proof. The solution $y$ of (11) can be written in the form $y(t)=z(t)-w(t)$, with

$$
z(t)=S(t) y_{0}+\int_{0}^{t} S(t-s) F(s) d s
$$

and

$$
w(t)=\int_{0}^{t} S(t-s)[a y+B \cdot \nabla y](s) d s .
$$

Since $y_{0} \in W^{2, p}(\Omega)$ with $p>N$ and $F \in L^{q}(Q)$ with $q>N+2$, it is not difficult to see that $z \in L^{\infty}\left(0, T ; W^{1, \infty}(\Omega)\right)$ and

$$
\|z\|_{L^{\infty}\left(0, T ; W^{1, \infty}\right)} \leq C\left\|y_{0}\right\|_{W^{2, p}}+\frac{C}{\alpha(q)} T^{\alpha(q)}\|F\|_{q} \leq e^{C_{q}(1+T)}\left(\left\|y_{0}\right\|_{W^{2, p}}+\|F\|_{q}\right),
$$

where

$$
\alpha(q)=\frac{q-(N+2)}{2(q-1)} .
$$

On the other hand, the usual Sobolev imbeddings give $y \in L^{\infty}\left(0, T ; L^{\gamma}(\Omega)\right)$ ( $\gamma$ is given in (76)). Moreover, we can write the following for all $t>0$ :

$$
\left\{\begin{aligned}
\|w(\cdot, t)\|_{W^{1, \gamma}} \leq & C \int_{0}^{t}(t-s)^{-1 / 2}\|(a y)(\cdot, s)\|_{L^{\gamma}} d s \\
& +C \int_{0}^{t}(t-s)^{-1 / 4}\|(B \cdot \nabla y)(\cdot, s)\|_{L^{r}} d s .
\end{aligned}\right.
$$

We can now apply Young's inequality to obtain

$$
\|w\|_{L^{\infty}\left(W^{1, \gamma}\right)} \leq C\left(T^{1 / 2}\|a\|_{\infty}+T^{1 / 4}\|B\|_{\infty}\right)\|y\|_{L^{\infty}\left(0, T ; W^{1, r}\right)} .
$$

This completes the proof of Lemma A.1.

We are now ready to prove (14). Since $y_{0} \in H_{0}^{1}(\Omega)$ and $F \in L^{2}(Q)$, the classical energy estimates give

$$
y \in L^{\infty}\left(0, T ; H_{0}^{1}(\Omega)\right) \cap L^{2}\left(0, T ; H^{2}(\Omega)\right),
$$

with

$$
\|y\|_{L^{\infty}\left(0, T ; H_{0}^{1}\right)}+\|y\|_{L^{2}\left(0, T ; H^{2}\right)} \leq e^{C\left(1+T+\left(T+T^{1 / 2}\right)\|a\|_{\infty}+T\|B\|_{\infty}^{2}\right)}\left(\left\|y_{0}\right\|_{W^{2, p}}+\|F\|_{q}\right) .
$$


We can now apply Lemma A.1 with $r=2$ and obtain $y \in L^{\infty}\left(0, T ; W^{1, r_{1}}(\Omega)\right)$, where

$$
\frac{1}{r_{1}}=\frac{1}{2}-\frac{1}{2 N}
$$

and

$$
\left\{\begin{aligned}
\|y\|_{L^{\infty}\left(0, T ; W^{\left.1, r_{1}\right)}\right.} \leq & e^{C(1+T)}\left(\left\|y_{0}\right\|_{W^{2, p}}+\|F\|_{q}\right) \\
& +e^{C\left(1+T^{1 / 2}\|B\|_{\infty}^{2}+T^{1 / 2}\|a\|_{\infty}\right)}\|y\|_{L^{\infty}\left(0, T ; H_{0}^{1}\right)}
\end{aligned}\right.
$$

Combining the last two inequalities, we obtain

$$
\|y\|_{L^{\infty}\left(0, T ; W^{1, r_{1}}\right)} \leq e^{C\left(1+T+\left(T+T^{1 / 2}\right)\|a\|_{\infty}+\left(T+T^{1 / 2}\right)\|B\|_{\infty}^{2}\right)}\left(\left\|y_{0}\right\|_{W^{2, p}}+\|F\|_{q}\right) .
$$

We can repeat this process for $i=2, \ldots, N$, with

$$
r_{i}=\left(\frac{1}{2}-\frac{i}{2 N}\right)^{-1} \quad \text { for } i \leq N-1 \quad \text { and } \quad r_{N}=\infty .
$$

Obviously, this leads to (14).

Acknowledgments. The authors thank the referees for their interesting comments and suggestions. In particular, they are indebted to referee no. 1 for having suggested the argument used in the proof of Theorem 1.2.

\section{REFERENCES}

[AB] S. Anita AND V. BARBU, Null controllability of nonlinear convective heat equation, ESAIM Control Optim. Calc. Var., 5 (2000), pp. 157-173.

[A] J.P. Aubin, L'Analyse non Linéaire et ses Motivations Économiques, Masson, Paris, 1984.

[B] V. BARBU, Exact controllability of the superlinear heat equation, Appl. Math. Optim., 42 (2000), pp. 73-89.

[CH] Th. Cazenave And A. Haraux, Équations d'évolution avec non-linéarité logarithmique, Ann. Fac. Sci. Toulouse, 2 (1980), pp. 21-51.

[FPZ] C. Fabre, J.P. Puel, and E. Zuazua, Approximate controllability of the semilinear heat equation, Proc. Roy. Soc. Edinburgh Sect. A, 125 (1995), pp. 31-61.

[F] E. FERnÁNDEZ-CARA, Null controllability of the semilinear heat equation, ESAIM Control Optim. Calc. Var., 2 (1997), pp. 87-107.

[FZ1] E. FERnÁNDEZ-CARA AND E. ZuAZUA, The cost of approximate controllability for heat equations: The linear case, Adv. Differential Equations, 5 (2000), pp. 465-514.

[FZ2] E. FernándeZ-CARA And E. ZuAZuA, Null and approximate controllability for weakly blowing up semilinear heat equations, Ann. Inst. H. Poincaré Anal. Non Linéaire, 17 (2000), pp. 583-616.

[FI] A. Fursikov and O. Yu. Imanuvilov, Controllability of Evolution Equations, Lecture Notes Ser. 34, Seoul National University, Korea, 1996.

[I] O. Yu. Imanuvilov, Controllability of parabolic equations, Mat. Sb., 186 (1995), pp. 102132.

[IY] O. Yu. Imanuvilov and M. Yamamoto, Carleman estimate for a parabolic equation in a Sobolev space of negative order and its applications, in Control of Nonlinear Distributed Parameter Systems, Lecture Notes in Pure and Appl. Math. 218, Dekker, New York, 2001, pp. 113-137.

[LSU] O.A. Ladyzenskaya, V.A. Solonnikov, and N.N. Uraltzeva, Linear and Quasilinear Equations of Parabolic Type, Nauka, Moscow, 1967.

[S] J. Simon, Compact sets in the spaces $L^{p}(0, T ; B)$, Ann. Mat. Pura Appl. (4), 146 (1987), pp. 65-96.

[Z1] E. ZuAZuA, Exact boundary controllability for the semilinear wave equation, in Nonlinear Partial Differential Equations and Their Applications, Vol. 10, H. Brezis and J.L. Lions, eds., Longman Scientific and Technical, Harlow, 1991, pp. 357-391.

[Z2] E. ZuAZUA, Approximate controllability for semilinear heat equations with globally Lipschitz nonlinearities, Control Cybernet., 28 (1999), pp. 665-683. 